\title{
II metodo Freedom Writers: Aiutare gli adolescenti a scrivere di sè e celebrare il cambiamento
}

\section{The Freedom Writers Method: Helping teens to write about themselves and celebrate the change}

\begin{abstract}
This article presents the experience of a teacher - Erin Gruwell - an English literature teacher at a school in Long Beach, Los Angeles (California). The teacher, proposing in school writing a personal diary at school, created significant conditions for change for the students. The educational experiences offered by Erin Gruwell spurred the motivation to write diaries. Doing this has opened up a reflective space on their hard life: the students have begun to think about a future of hope. Of particular significance was the experience of „Toast for Change”, which marked a new beginning in the lives of these students.
\end{abstract}

\section{Keywords}

Write, autobiography, availability, diary, to change the future, hope, motivation. 
«You are the promise»

(«Voi siete la promessa»)

Erin Gruwell ai Freedom Writers

10 Agosto 2014, Art Theatre di Long Beach

\section{Introduzione}

L'autobiografia è un modo di farsi carico di sé, di prendersi a cuore, è ricerca sociale, è autoanalisi e maturazione. Può essere una sfida per l'adulto che vuole aiutare l'adolescente, spesso spavaldo ma dentro molto fragile, a superare i compiti di sviluppo della propria età. L'atto dello scrivere potrebbe diventare quella distanza che consente a questo adolescente di pensare il "pensato", quell'incessante bisogno di dare ragione dei suoi tumulti emozionali. Scrivere è fermarsi, provare a balbettare risposte circa il proprio essere al mondo, il progetto personale; scrivere è passare nel buio del non conosciuto, del misterioso, per arrivare alla consapevolezza. L'applicazione degli strumenti narrativi nel lavoro educativo e formativo con gli adolescenti, anche all'interno del contesto scolastico, ha conosciuto negli ultimi anni una evoluzione crescente. Il sé narrativo riguarda il processo di costruzione del sé determinante per la disponibilità all'apprendimento. Per gli studenti che scelgono di scrivere un diario è rilevante l'importanza della micrologia del quotidiano, del racconto di fatti apparentemente insignificanti, della ricostruzione di frammenti incompiuti di vita che consentono la maturazione dell'attenzione che può diventare, come indica W. Benjamin nella sua analisi dello stile narrativo di Kafka, "la preghiera naturale dell'anima".

\section{Scrivere e apprendere di sé: processi trasformativi attraverso la scrittura del diario}

La costruzione dello scenario teorico di fondo dello scrivere di sé si riferisce principalmente ad autori come J. Bruner, D. Demetrio, L. Mortari. ${ }^{2}$

${ }^{1}$ Cfr. J. Benjamin, Angelus Novus, (tr. it. di R. Solmi), Einaudi, Torino 1962 e 1995, 299.

${ }^{2}$ Cfr. J. Bruner, In search of mind: essay in autobiography, New York, Harper \& Row 1983 (tr. It., Autobiografia. Alla ricerca della mente, Roma, Armando 1984); D. Demetrio, Raccontarsi. L’autobiografia come cura di sé, Milano, Raffaello Cortina 1996; ID, Pedagogia della memoria. Per sé, per gli altri, Roma, Meltemi 1998; ID., L'educazione interiore. Introduzione alla 
Lo scrivere è l'ultimo atto di un interminabile processo interno che va accompagnato da adulti significativi: imparare a maneggiare il senso del vuoto, imparare a scrivere delle proprie speranze e delle proprie disavventure. Dentro lo scrivere cè la lettura di storie, il leggere biografie, entrare in contatto con esperienze che sviluppano disponibilità al cambiamento. Il groviglio del sentire può essere "mentalizzato" grazie a questa incessante operazione del trovare le proprie parole per raccontarsi. Il bisogno esplorativo dell'adolescente, può essere tracciato da questi segnali dell'alfabeto, da parole che hanno corpo, parole che trasformano eventi in esperienze. Scrivere è imparare a metariflettere, imparare ad apprendere dall'esperienza, da quel groviglio diventato parola perché fatto rinascere dalla scrittura. Faticoso e incessante prendere posizione, sviluppare il proprio punto di vista per non nascondersi nei meandri del collettivo, dell'indifferenziato. In questo percorso, l'adolescente ha bisogno di essere accompagnato e i luoghi educativi possono diventare la piazza della memoria, il luogo dell'imparare a scrivere, il luogo del prendersi del tempo, il luogo della lentezza. Scrivere può aumentare lo spazio della libertà interiore, lo spazio del rispetto delle differenze.

Promuovere questi spazi educativi come luoghi produttori di un'esperienza indelebile nella vita di chi li abita è come un "trampolino di lancio" per un futuro all'insegna di consapevolezza e di intelligenza autobiografica, come persone facenti parte di una comunità civile pensante. Il sapere, a queste condizioni, dà sapore alla vita. In questo discorso, rientrano i luoghi educativi che sviluppano comprensione profonda: la scuola, ma anche comunità religiose, territori formativi. In questa dinamica autobiografica, il processo di apprendimento è un incontro con la biografia del testimone autorevole, che sia Dante o Ungaretti oppure Einstein, un incontro con la biografia - vita dell'adolescente - e un incontro con la storia dell'educatore che, stando sempre alla soglia, non affronta mai di petto il sentire dell'adolescente-studente ma lo mette in relazione significativa con il testimone autorevole proposto. Grazie a questa sua consapevole presenza, l'adulto potrebbe aiutare l'adolescente a costruirsi persona, a diventare disponibile ai cambiamenti. Accompagnare l'adolescente-studente potrà significare allora mettersi nella condizione di scrivere la propria autobiografia oppure provare

pedagogia introspettiva, Milano, Rcs Libri 2000; ID., Ricordare a scuola. Fare memoria e didattica autobiografica, Roma-Bari, Laterza 2003; ID., La scrittura clinica. Consulenza autobiografica e fragilità esistenziali, Milano, Raffaello Cortina 2008; L. Mortari, Aver cura della vita della mente, Firenze, La Nuova Italia 2002; ID., Apprendere dallesperienza. Il pensare riflessivo nella formazione, Roma, Carocci 2003; ID., La pratica dell'aver cura, Mondadori, Milano 2006; ID., Aver cura di sé, Mondadori, Milano 2009. 
a dare parola a quel evento misterioso e bello che è il processo dell'apprendere. Ognuno con la sua penna sviluppa il personale processo di individuazione, ognuno con il suo modo particolare di premere la penna tra le mani compie quel faticoso travaglio che lo distanzia dalle figure significative, quel misterioso cammino di separazione e individuazione. Con la penna, con l'atto dello scrivere si risveglia il desiderio di osservare il mondo attorno a sé. Noi sappiamo quanto sia importante per lo studente il gruppo dei pari: scrivere in classe o in comunità e condividere può risultate utile per sconfiggere i fantasmi della vergogna e la paura di sbagliare. Lo scrivere può risultare una particolarissima strada di educazione alla libertà e al rispetto delle differenze.

\section{Erin Gruwell: L'esperienza della scrittura del diario come strada per il cambiamento}

Erin Gruwell ${ }^{3}$ si è diplomata alla Bonita High School, a La Verne, California. Poi ha conseguito un Baccalaureato al College of Education dell'University of California di Irvine, dove ha ricevuto lodi e premi tra gli studenti che si sono distinti nella loro laurea. Ha raggiunto poi un diploma di Master alla California State University di Long Beach, dove è stata onorata come studentessa che si è distinta nel Dipartimento dell'Educazione con cui collaborava.

Nel 1995 iniziò l'avventura con gli adolescenti della classe 203 della Wilson High School; in questa classe multiculturale, promuovendo una filosofia educativa che valorizza e promuove la diversità, la Gruwell trasformò la vita dei suoi studenti. Li ha incoraggiati a ripensare i rigidi pregiudizi su loro stessi e sugli altri, a rivedere le loro decisioni quotidiane e, in ultima analisi, ri-tracciare il loro futuro. Questi ragazzi, con il supporto della Gruwell, attraverso la scrittura del diario sulla storia della propria vita, hanno creato condizioni per diventare catalizzatori del cambiamento.

Scrivere: una formazione personale generatrice di cambiamenti indotti dall'imparare, si potenziano le capacità cognitive, la penna ed il cervello si alleano per mentalizzare, per simbolizzare la propria esperienza, per connotarla di senso; un esercizio che unisce corpo e mente. Scrivere è questo cammino di unificazione tra il pensare, il sentire, l'agire. Nelle storie di questi studenti

${ }^{3}$ Cfr. D. Stevens Comparing the movie Freedom Writers to the real life of Erin Gruwell, Edited by Dakota Steven From High Quality, Wikipedia Articles 2014. 
della classe 203, i Freedom Writers, cè la voglia di reagire alla forza oscura della depressione, della sfiducia in se stessi, dello smarrimento più devastante. Scrivere: una tecnologia umile anzi povera sempre a portata "della mano" con mille combinazioni creative. ${ }^{4}$

«Il male, scrivendo, lo avvolgiamo in una rete di parole; come in un bozzolo che così ci portiamo appresso per svolgerlo (rileggerlo e sopportarne finalmente la natura) in un tempo diverso. Nella consapevolezza che il non averlo espulso rimuovendolo vanamente nell'inconscio, ma avendolo aggredito con la penna, lo "addomestica"». ${ }^{5}$

La scrittura non libera ma dà senso. Il lettuccio delle nostre disattenzioni lo porteremo sempre con noi. Gli alunni della 203 hanno pianificato attraverso la scrittura del loro diario un passaggio: dalla cittadella del proprio ego alla piazza, all'attenzione a ciò che succedeva intorno a loro. Da persone imperfette, consapevoli dei loro limiti, hanno "marciato" prendendo contatto con il loro "marcio" per pensare ad un mondo migliore. Il bisogno fondamentale di ogni studente che entra nel mondo-aula scolastica è quello di sentirsi confermato come persona, incessantemente a livello quasi inconsapevole si porta nel sottofondo delle sue attese una domanda come sussurrata allorecchio: "Mi sentirò bene qui?". Per realizzare questo obiettivo, lo studente entrando in un'aula scolastica ha bisogno di avere determinate consapevolezze:

- "Qui dentro sono accettato e merito accettazione".

- "Mi sento al sicuro qui, così come sono".

- "Qui le persone mi ascoltano".

- "Le persone sanno come sto lavorando ed il mio lavoro conta".

- "I miei interessi e le mie idee sono riconosciute e tenute presenti".

" "Qui le persone credono in me".6

Erin Gruwell sembra aver creato le condizioni di una accettazione incondizionata che ha permesso a questi studenti di mischiare il loro sangue con l'inchiostro, scrivere senza vergogna la propria storia. Nell'aula 203 gli studenti iniziano a sentirsi persone, lavorano perché insieme si valorizzi l'unicità, l'auto-espressione, scrivono lettere anche con la paura di sbagliare, di essere

${ }^{4}$ Cfr. D. Demetrio, Raccontarsi. L’autobiografia come cura di sé.

${ }^{5}$ D. Demetrio, La scrittura clinica, XII.

${ }^{6}$ Cfr. C. A. Tomlison, Fulfilling the Promise of the Differentiated Classroom. Strategies and Tools for Responsive Teaching, USA, Association Supervision for Curriculum Development 2003 (tr. it., Adempiere la promessa di una classe differenziata. Strategie e strumenti per un insegnamento attento alla diversità, Las, Roma 2006, 34). 
sgrammaticati. Ad un certo punto del percorso, quando Erin ha tracciato strade per un reale coinvolgimento dei suoi studenti, proprio nel momento del "Brindisi per il cambiamento", propone ai suoi studenti di scrivere le loro storie. Un adulto che protegge le loro storie fragili e valorizza i loro talenti seppelliti nella rabbia. Erin invita i suoi studenti a scrivere la loro storia. Ecco cosa racconta: «I diari provvederemo a metterli in un posto sicuro per diventare appassionati scrittori, comunicando le nostre personali storie, le nostre personali intuizioni. Dal momento in cui essi cominciarono a mettere giù i loro pensieri e sentimenti, la motivazione fiorì. Improvvisamente trovarono un forum per l'autoespressione e un posto dove potevano sentirsi valutati e validati. I miei studenti furono ispirati a scrivere lettere a Miep Gies, la donna coraggiosa che nascose Anna Frank, Zlata Filippovic, la giovane autrice che scrisse "Il diario di Zlata una vita di infanzia a Sarajevo". Quando Miep Gies disse ai miei studenti di stare sicuri che la morte di Anna non è stata invano, essi capirono il suo messaggio: che scrivere e raccontare storie ha il potere di cambiare il mondo. Seguendo i passi dell'adolescenza straordinaria di Anna e Zlata, ${ }^{7}$ i miei studenti usarono i loro personali diari per condividere le loro esperienze di perdita, di privazioni o sofferenza e discriminazione. Come un bambino io ho fatto vedere ai miei studenti un documentario sui Freedom Riders, gli attivisti dei diritti civili i quali, attraversarono in autobus il sud nel 1961 insieme ai bianchi. Il coraggio dei Freedom Rides ispirò la mia classe ad adottare il nome "Freedom Writers" per descrivere la determinazione dei miei studenti a usare i loro diari per raccontare il razzismo e l'intolleranza che li accerchiava, li circondava. Per celebrare la loro nuova identità, i Freedom Writers seguirono i passi dei Freedom Rides e intrapresero un viaggio per Washington; in un simbolico tributo ai loro omonimi essi consegnarono una copia rilegata dei loro diari preferiti a Richard Riley, il Segretario dell'Educazione degli Stati Uniti. I FW ricevettero il premio dello Spirito di Anna Frank per il loro impegno a combattere discriminazione, razzismo e violenza. Essi anche dedicarono molte ore a redigere i loro diari e furono ricompensati con un contratto pubblico di trasformare i loro diari di classe che sarebbero diventati un best seller: "The Freedom Writers Diary: come un insegnante e 150 adolescenti usarono scrivere per cambiare loro stessi ed il mondo intorno a loro"». ${ }^{8}$

${ }^{7}$ Si tratta dei diari di Anna Frank e Zlata Filippovic; quest'ultima ha scritto nel periodo dell'assedio di Sarajevo, avvenuto durante la guerra in Bosnia ed Erzegovina, che è stato il più lungo assedio nella storia bellica moderna, protrattosi dal 5 aprile 1992 al 29 febbraio 1996.

8 Testo tradotto da The Freedom Writers Foundation - E. Gruwell, The Freedom Writers Diary Teacher's Guide, York, Broadway Books 1999, 2. 
Con l'esperienza del Brindisi per il cambiamento, che avremo modo di descrivere più avanti in questo articolo, l'insegnante Gruwell invita i suoi studenti della 203 a scrivere. Nascono 150 storie di cambiamento di questi giovani studenti che scelsero il nome di Freedom Writers; per esigenze di sintesi ne evidenziamo solo alcune per sottolineare che proprio latto dello scrivere della propria esperienza crea passaggi: dall'atto riflessivo dello scrivere all'apprendimento come cambiamento di punti di vista, come nuove strategie comportamentali per risolvere i problemi, come nuovo modo di affrontare i problemi, intesi come uno stimolo per il cambiamento.

Louverture di questo diario dei Freedom Writers, che è stato poi pubblicato con un grande successo negli Stati Uniti fino alla realizzazione di un film legato a queste storie di vita, ${ }^{9}$ sono i pensieri dell'insegnante Erin Gruwell, che noi abbiamo a disposizione grazie alla sua pratica riflessiva quotidiana. Un invito a coltivare un tempo dell'interiorità perché dal docente-educatore in prima persona possa iniziare il proprio cambiamento. Gli stralci presi da questo diario sono corredati da domande sviluppate dalla stessa signora Gruwell nella Guida relativa al Metodo educativo Freedom Writers. ${ }^{10}$

Dal Diario 1. ${ }^{\text {a }}$ "Voce 1: Sig.ra Gruwell

Caro diario domani mattina, il mio viaggio come insegnante di inglese inizia ufficialmente. Dal momento che le prime impressioni sono così importanti, mi chiedo che cosa i miei studenti penseranno di me. Penseranno che sono adeguata oppure che sono troppo giovane? (oppure una giovane che veste bene e viene da una scuola bene!). O peggio ancora, che io sono troppo giovane per essere preso sul serio? Forse farò scrivere le loro aspettative che hanno nei miei confronti e nei confronti della classe. Anche se ho trascorso lo scorso anno come insegnante studente (tirocinante) presso la Wilson, sto ancora imparando a conoscere la città".

Domanda della Guida: "La Signora Gruwell è frustrata dalle etichette date ai suoi studenti. Mettetevi nei suoi panni. Che cosa ms. G. ha fatto nei primi giorni di scuola per mostrare i suoi studenti che lei non crede in queste etichette?"b

a Cfr. The Freedom Writers with E. Gruwell, The Freedom Writers Diary. How a Teacher and 150 Teens Used Writing to Change Themselves and the World Around Them, cit., 1-5.

b Testo tradotto da The Freedom Writers Foundation - E. Gruwell, The Freedom Writers Diary Teacher's Guide, cit., 217.

9 Cfr. The Freedom Writers - Gruwell E., The Freedom Writers Diary: How a Teacher and 150 Teens Used Writing to Change Themselves and the World Around Them, cit.; Film: Freedom Writers. Le loro storie. Le loro parole. Il loro futuro, Swank Hilary (produttore), Lagravenese Richard, California, Los Angeles, A-S Paramount Picture 2006.

${ }^{10}$ Cfr. The Freedom Writers Foundation - Gruwell E., The Freedom Writers Diary Teacher's Guide, New York, Broadway Books 2007. 
In riferimento al Diario $1{ }^{\text {a }}$ - Primo giorno di scuola. Le etichette.

In questo diario lo studente, nei primi giorni di scuola, passa in rassegna con etichette e soprannomi i compagni di classe. Il primo giorno questo studente passa sotto giudizio Miss G. e gli altri studenti della classe senza aspettare di arrivare a conoscerli prima.

Domanda della Guida: Perché la gente si etichetta l'un l'altro? Come ti sentiresti se tu fossi seduto in quella classe? ${ }^{\mathrm{b}}$

a Cfr. The Freedom Writers with E. Gruwell, The Freedom Writers Diary, cit., 1-5.

b Testo tradotto da The Freedom Writers Foundation - Gruwell E., The Freedom Writers Diary Teacher's Guide, cit., 217.

Uguali ma separati: l'intolleranza razziale in particolare negli Stati Uniti si è manifestata nelle forme concrete delle scuole con classi distinte in bianchi e neri. Lo scrittore del diario 2 racconta la presenza di queste forme di separazione guardando le varie gang sedute insieme per etnia durante la mensa scolastica. E' il 1994 !

In riferimento al Diario $2 .^{\mathrm{a}}$ - Segregazione razziale a scuola.

Questo studente descrive come avviene di fatto la segregazione nella scuola. Avviene nel momento del pranzo. Quando ogni etnia si mette insieme.

Domanda della Guida: Ma cosa succederebbe se un estraneo entrasse nei confini di un'altra comunità? Quali gli effetti positivi? Quali gli effetti negativi?b

a Cfr. The Freedom Writers with E. Gruwell, The Freedom Writers Diary, cit., 12-14.

b Testo tradotto da The Freedom Writers Foundation - E. Gruwell, Teacher's Guide, cit., 217.

Nell'ambiente di Los Angeles, a Long Beach, può capitare di sentirsi sicuro tra coetanei solo se si ha una pistola, convivere con il freddo dell'arma appiccicata in ogni momento al proprio corpo.

In riferimento al Diario $5{ }^{\text {a }}$ - L'acquisto di una pistola.

Questo FW descrive come acquistando una pistola gli ha fornito un senso di forza e di protezione anche se era pericoloso.

Domanda della Guida: Pensate ai problemi che dovete affrontare nel vostro vivere quotidiano. Descrivi quello che offre diritto e protezione. È qualcosa di fisico o una parte della tua personalità? ${ }^{\mathrm{b}}$

${ }^{a}$ Cfr. The Freedom Writers with E. Gruwell, The Freedom Writers Diary, cit., 12-14.

b Testo tradotto da The Freedom Writers Foundation - E. Gruwell, Teacher's Guide, cit., 218.

Questi brevi stralci fanno capire da quale contesto l'educatrice Erin Gruwell è partita per portare i suoi studenti al cambiamento di vita. Ha tenuto conto dell'esperienza dei suoi studenti, si è preoccupata di tenere presente oltre che 
i prerequisiti della disciplina come ogni insegnante anche delle esperienze esistenziali di questi adolescenti.

\section{Brindisi per il cambiamento: generare speranza a partire dai gesti del presente}

Il "Brindisi per il cambiamento" è una delle più significative esperienze educative proposto dalla signora Erin Gruwell, è l'inizio del cambiamento che sarebbe dovuto essere testimoniato dalla scrittura quotidiana del diario.

Questa celebrazione rappresenta la promessa, il passaggio, la volontà di cambiare, la testimonianza di fronte alla propria comunità-classe di usare le stesse avversità per diventare una persona migliore per "accendere una luce". Con il Brindisi per il cambiamento si inizia a vivere per una seconda volta, tutti i no e le limitazioni della prima vita di questi adolescenti sono messi da parte.

Presentiamo il modo in cui la stessa Gruwell ha proposto di vivere questo "brindisi".

«Obiettivo: Il brindisi per il cambiamento fu un momento centrale nel diario dei Freedom Writers. L’obiettivo di questa attività è riconoscere le passate esperienze dei tuoi studenti, mentre offri loro una opportunità per cambiare la direzione delle loro vite. Gli studenti spesso credono che le scelte passate e i loro risultati scolastici condizioneranno i loro insegnanti. Il brindisi per il cambiamento è un modo simbolico di comunicare ai tuoi studenti che quanto loro hanno fatto prima passa nella tua classe ma non conta più, non ha peso, nella valutazione. Questa attività dovrebbe essere considerata un nuovo inizio per i tuoi studenti, uno che aiuterà loro a costruire la loro strada per il successo».

Il diario tenuto giornalmente dalla docente E. Gruwell ci consente di comprendere bene il processo che ha fatto nascere l'intuizione di presentare il Brindisi per il cambiamento. Ecco cosa scrive Gruwell:

«Dalla storia della 203: Nel mezzo della notte dei miei studenti, ho avuto una intuizione pazzesca che ha rappresentato una "seconda possibilità" per i miei studenti. Il brindisi per il cambiamento ha consentito ai miei studenti di fare una scelta positiva per la loro vita, ogni studente si è guadagnato rispetto per se stesso e per la sua classe. L'esperienza ha fornito un ambiente sicuro per condividere emozioni e sviluppare coesione nella classe.

La docente E. Gruwell ho intuito loccasione giusta per celebrare questo importante rito, gli studenti erano motivati a fare una scelta importante, non 
erano più presenti conflitti di gruppo e mancanza di rispetto all'interno della classe; oramai le attività della Gruwell avevano creato interdipendenza positiva, bisogno di crescere insieme, bisogno di confrontarsi sui propri obiettivi di vita. Ecco i consigli che la docente scrive nella sua Guida Didattica:

"Consigli di Miss G.: Come insegnante dei tuoi studenti, solo tu puoi sapere quando è opportuno fare l'esperienza del "Brindisi per il cambiamento". Per alcuni studenti, il brindisi è una grande via che indica i "passaggi" che loro fanno nella scrittura, è la via verso il successo. Per altri studenti risulta significativo durante l'attività del "Dre potere agli studenti" come culmine delle attività. Quando senti che i tuoi studenti sono pronti, prepara la classe: un rituale che li faccia sentire persone speciali. Siate preparati per questa attività in modo da suscitare intense emozioni nei vostri studenti».

Il rituale ha i suoi momenti fondamentali descritti con molto senso della cura e attenzione dalla docente Gruwell:

"Quello che vi serve: Bicchieri di plastica per lo champagne. Sidro frizzante di mela. Tovaglia di carta e asciugamani. Il grafico visivo del "brindisi per il cambiamento".

Il processo:

Step 1: Prepara un bicchiere di sidro frizzante per ogni studente.

Step 2: Metti alla parete la cattedra, cè una zona grande attorno alla quale i tuoi studenti possono stare in circolo.

Step 3: Come i tuoi studenti arrivano, chiedi loro di lasciare i loro zaini attorno al muro e poi fare un circolo nella stanza.

Step 4: Chiedi ad un paio di volenterosi di aiutarti a passare i bicchieri al gruppo. Chiedi ai tuoi studenti di raccontare i loro cambiamenti. Dopo che tutti hanno raccontato, allora insieme si brinda, si beve!

Step 5: Introduci il brindisi per il cambiamento e spiega loro che si tratta di iniziare cambiamenti nella propria vita.

Step 6: Ognuno degli studenti che si trova in cerchio dice che cosa vorrebbe cambiare della propria vita. Anche il docente fa lo stesso. Gli studenti apprezzeranno la sincerità del docente.

Step 7: Una volta che tutti hanno condiviso le narrazioni, gli studenti alzano i bicchieri per il brindisi del cambiamento.»

Mentre si condivide la narrazione la docente scrive i sentimenti che emergono dall'esperienza del gruppo, è necessario visualizzare il percorso per dare la possibilità alla comunità degli adolescenti che spesso fanno fatica a parlarsi di sperimentare il clima di accoglienza e accettazione incondizionata. 
"Grafico visuale: Il grafico visivo del brindisi del cambiamento serve per registrare le risonanze emotive, serve per far diventare questo fatto una esperienza significativa».

La parola nuova che il gruppo ha scoperto in questa esperienza di Brindisi per il cambiamento è epifania, in questo contesto possiamo parlare di rivelazione intesa come qualcosa che non ci si aspetta che avviene, un evento inatteso. E' il sentimento di disperazione che sciogliendosi apre le porte alla fiducia per un futuro migliore.

«Vocabolario: Epifania».

Il cambiamento è contornato da pochissime parole e molti gesti, molte azioni, tanta progettualità e riflessione sui modi concreti di attuare i passaggi dalla cittadella dell'ego alla cittadella della relazione e della cura dellaltro.

«Valutazione: Discuti con i tuoi studenti che cosè un'epifania e descrivi come per qualcuno, l'esperienza del cambiamento, il brindisi per il cambiamento è una vera rivelazione. Dopo di questo, gli studenti scrivono su un cartellone la parola chiave che esprime la loro epifania, la loro rivelazione, il loro cambiamento. Il docente dovrebbe incontrare ogni studente per aiutarli a pensare ad un piano di azioni per attivare il cambiamento. Discutere di come il brindisi sia un potente rituale condiviso da molte culture e di solito associato all'onore, impegni, buona salute, auguri e celebrazioni. Mentre discutono dei loro rituali possono mangiare qualcosa».

Docenti come testimoni del cambiamento, come accompagnatori silenziosi, persone che vivono il comandamento del cambiare come conversione quotidiana: sii il cambiamento che vuoi che avvenga negli altri. Questa è la parola importante che la docente Gruwell e tanti altri docenti hanno praticato e praticano con mani e cuore.

Per approfondire: Altra possibilità è quella di chiedere alla classe nel suo complesso di identificare i problemi della propria comunità e vedere che cosa piacerebbe loro cambiare. Questo può diventare un progetto di apprendimento per la classe. Gli studenti dovrebbero creare un piano di azione per attuare questo cambiamento.

Il Feedback dei Freedom Writers: "Il brindisi per il cambiamento è stato un momento decisivo per me. Alzando un bicchiere di plastica, sembra un piccolo passo verso il cambiamento, ma mi ha aiutato a definire i miei obiettivi e credo che ho potuto raggiungerli. Il giorno in cui abbiamo brindato è stato anche il giorno in cui tutti noi cominciammo ad essere reali con l'altro. Abbiamo condiviso qualcosa di speciale e questo legò noi tutti”. 
Diario 31: "Ho avuto una seconda possibilità di cambiare la mia vita in meglio. Ringrazio Dio che ha mandato un angelo (Gruwell) per darmi questa possibilità di cambiare". ${ }^{11}$

Conversazioni con i docenti: Se si trova il momento giusto questa è una esperienza di autentico cambiamento». ${ }^{12}$

\section{Conclusione}

In ogni ambiente educativo, che sia la scuola o una comunità religiosa, sembra necessario dunque ritualizzare gli eventi del cambiamento. La scrittura del diario e il brindisi per il cambiamento, presentati dalla Gruwell, sono una possibile via di educazione alla libertà, alla valorizzazione delle differenze e una via per preparare progetti futuri intessuti di speranza. La testimonianza della docente Gruwell e dei Freedom Writers rappresenta una delle tante strade che dicono la necessità di trasformare le parole parlate in parole progetto, parole vissute. L'esperienza educativa dei Freedom Writers rimane una strada molto concreta da esplorare e praticare in altri contesti oltre che la scuola.

\section{Bibliography}

Benjamin J., Angelus Novus, (tr. it. di R. Solmi), Einaudi, Torino 1962 e 1995.

Bruner J., In search of mind: essay in autobiography, New York, Harper \& Row 1983 (tr. It., Autobiografia. Alla ricerca della mente, Roma, Armando 1984).

Cursio G., Il Metodo Freedom Writers: sviluppare cambiamento negli studenti, Estratto tesi di Dottorato n. 934, Università Pontificia Salesiana, Roma 2015.

Demetrio D., Raccontarsi. L'autobiografia come cura di sé, Milano, Raffaello Cortina 1996.

Demetrio D., Pedagogia della memoria. Per sé, per gli altri, Roma, Meltemi 1998.

Demetrio D., L'educazione interiore. Introduzione alla pedagogia introspettiva, Milano, Rcs Libri 2000.

Demetrio D., Ricordare a scuola. Fare memoria e didattica autobiografica, Roma-Bari, Laterza 2003.

${ }^{11}$ Qui ci si riferisce a The Freedom Writers - E. Gruwell, The Freedom Writers Diary, cit., $61-62$.

12 Testo tradotto da The Freedom Writers Foundation - E. Gruwell, The Freedom Writers Diary Teacher's Guide, cit., 63-66. 
ID., La scrittura clinica. Consulenza autobiografica e fragilità esistenziali, Milano, Raffaello Cortina 2008.

Mortari L., Aver cura della vita della mente, Firenze, La Nuova Italia 2002.

Mortari L., Apprendere dall'esperienza. Il pensare riflessivo nella formazione, Roma, Carocci 2003.

Mortari L., La pratica dell'aver cura, Mondadori, Milano 2006.

Mortari L., Aver cura di sé, Mondadori, Milano 2009.

Stevens D., Comparing the movie Freedom Writers to the real life of Erin Gruwell, Edited by Dakota Steven From High Quality, Wikipedia Articles 2014.

The Freedom Foundation - E. Gruwell, Writers The Freedom Writers Diary Teacher's Guide, York, Broadway Books 1999.

The Freedom Writers Foundation - Gruwell E., The Freedom Writers Diary Teacher's Guide, New York, Broadway Books 2007.

Tomlison C. A., Fulfilling the Promise of the Differentiated Classroom. Strategies and Tools for Responsive Teaching, USA, Association Supervision for Curriculum Development 2003 (tr. it., Adempiere la promessa di una classe differenziata. Strategie e strumenti per un insegnamento attento alla diversità, LAS, ROMA, 2006).

\section{Filmography}

Freedom Writers. Le loro storie. Le loro parole. Il loro futuro, Swank Hilary (produttore), Lagravenese Richard, California, Los Angeles, A-S Paramount Picture 2006. 